\title{
Miniaturization of Josephson Junctions for Digital Superconducting Circuits
}

\author{
I.I. Soloviev®, ${ }^{1,2,3, *}$ S.V. Bakurskiy, ${ }^{1,3,4}$ V.I. Ruzhickiy ${ }^{1,2,3}$ N.V. Klenov®,$^{1,2,3}$ M.Yu. Kupriyanov® ${ }^{1}$ \\ A.A. Golubov, ${ }^{4,5, \dagger}$ O.V. Skryabina ${ }^{1,4,6}$ and V.S. Stolyarov ${ }^{3,4}$ \\ ${ }^{1}$ Lomonosov Moscow State University Skobeltsyn Institute of Nuclear Physics, Moscow 119991, Russia \\ ${ }^{2}$ Lomonosov Moscow State University, Physics Department, Moscow 119991, Russia \\ ${ }^{3}$ Dukhov All-Russia Research Institute of Automatics, Moscow 101000, Russia \\ ${ }^{4}$ Centre for Advanced Mesoscience and Nanotechnology, Moscow Institute of Physics and Technology, State \\ University, Dolgoprudny, Moscow region 141700, Russia \\ ${ }^{5}$ Faculty of Science and Technology and MESA+ Institute of Nanotechnology, Enschede 7500 AE, Netherlands \\ ${ }^{6}$ Institute of Solid State Physics RAS, Chernogolovka 142432, Russia
}

(Received 28 March 2021; revised 20 July 2021; accepted 27 September 2021; published 29 October 2021)

\begin{abstract}
In this work, we briefly overview various options for Josephson junctions, which should be scalable down to nanometer range for utilization in nanoscale digital superconducting technology. Such junctions should possess high values of critical current, $I_{c}$, and normal state resistance, $R_{N}$. Another requirement is the high reproducibility of the junction parameters across a wafer in a fabrication process. We argue that superconductor-normal metal-superconductor $(S N-N-N S)$ Josephson junction of "variable thickness bridge" geometry is a promising choice to meet these requirements. Theoretical analysis of the $S N-N$ $N S$ junction is performed in the case where the distance between the $S$ electrodes is comparable to the coherence length of the $N$ material. The restriction on the junction geometrical parameters providing the existence of superconductivity in the $S$ electrodes is derived for the current flowing through the junction of an order of $I_{c}$. The junction heating, as well as available mechanisms for the heat removal, is analyzed. The obtained results show that a $S N-N-N S$ junction with a high (submillivolt) value of $I_{c} R_{N}$ product can be fabricated from a broadly utilized combination of materials like $\mathrm{Nb} / \mathrm{Cu}$ using well-established technological processes. The junction area can be scaled down to that of semiconductor transistors fabricated in the frame of a $40-\mathrm{nm}$ process.
\end{abstract}

DOI: 10.1103/PhysRevApplied.16.044060

\section{INTRODUCTION}

The promised end of Moore's law [1] brings attention to the so-called "beyond CMOS" technologies. One of them is the superconductor technology distinguished by high energy efficiency and high clock frequencies [2-5]. It is considered to be especially suitable for "cold electronics" operating between room temperature and the temperature of cryogenic payloads like quantum computers, quantum internet, or scalable sensors [6]. Since the 1980s, superconducting circuits have been consistently developed reaching a fairly mature level at the end of the 1990s, showing an implementation of digital and mixed-signal devices [7-9].

However, further progress has slowed down. The main reason was the low integration density. It caused, e.g., implementations of superconducting memory to be of impractical storage capacity [10] that in turn impeded the realization of superconducting processors. Nevertheless, a decade later the researches were whipped up by US

\footnotetext{
*isol@phys.msu.ru

†a.a.golubov@utwente.nl
}

government investments aimed at the development of a notional prototype of a superconducting computer [11]. Its purpose was to demonstrate a prospective application of superconductor technology in the field of supercomputing. Unfortunately, along with significant overall progress in design and fabrication, we have to admit that the functional density of superconducting circuits is still quite low. The recently demonstrated benchmark circuits for the modern state-of the-art $150 \mathrm{~nm}$ of Massachusetts Institute of Technology Lincoln Laboratory (MIT LL) processes are the shift registers with $1.3 \times 10^{7}$ Josephson junctions $(\mathrm{JJs})$ per square centimeter circuit density [12]. This can be compared with the achieved approximately $1.3 \times 10^{10} \mathrm{Tr} / \mathrm{cm}^{2}$ density of transistors in the most advanced 5 -nm process node of the MOSFET technology.

One of the most difficult puzzles of the scaling is the reduction of the size of the Josephson junction [3], which is a nonlinear element of superconducting circuits. In this paper, we examine the most common types of Josephson junctions on their scalability. We argue that a planar $S N$ $N$-NS junction ( $S$ stays for a superconductor, and $N$ for a normal metal) with variable-thickness bridge geometry 
is promising for miniaturization. We calculate temperature dependencies of the supercurrent and the characteristic voltage of the $S N-N-N S$ junction for various transparencies of the $S-N$ interface. Further, we consider limitations on the junction critical current providing stable superconducting state in the $S$ electrodes. We also discuss the heat balance in the $S N-N-N S$ structure and estimate the heating during junction operation. Finally, we discuss the achievable range of areas of the studied structure and possible ways of its fabrication.

\section{SCALABILITY OF JOSEPHSON JUNCTIONS}

A workhorse of digital superconductor technology is a sandwich superconductor-insulator-superconductor ( $S$ - $I$ S) $\mathrm{Nb} / \mathrm{Al}-\mathrm{AlO}_{x} / \mathrm{Nb}$ Josephson junction. Due to the good wetting of $\mathrm{Nb}$ with $\mathrm{Al}$, one can obtain a high uniformity of barrier transparency, which provides just a few percent of the technological spread [13] of its parameters: critical current, $I_{c}$, and normal state resistance, $R_{N}$. The routinely achieved critical-current density of the junctions, $j_{c}=0.1 \mathrm{~mA} / \mu \mathrm{m}^{2}$, corresponds to a small insulator thickness still providing the homogeneous $S-I$ boundary. The critical current of Josephson junction should exceed the noise current, $I_{N}=\left(2 \pi / \Phi_{0}\right) k_{B} T$ (where $k_{B}$ is the Boltzmann constant, $T$ is the temperature, $\Phi_{0}=\pi \hbar / e$ is the flux quantum, $\hbar$ is the reduced Planck constant, and $e$ is the electron charge), by about 3 orders of magnitude for low enough bit error rate. At the same time, the energy dissipation during junction switching is proportional to the critical current, $E_{J} \approx I_{c} \Phi_{0}$, that makes its high values undesirable. For standardly used helium temperature, $T=4.2 \mathrm{~K}$, it turns to $I_{c} \approx 0.1 \mathrm{~mA}$, and the area of the junction, $a=I_{c} / j_{c} \approx 1 \mu \mathrm{m}^{2}$.

Since the sandwich type of tunnel Josephson junction possesses relatively high self-capacitance, $c \geq 60 \mathrm{fF} / \mu \mathrm{m}^{2}$, the resistive shunt, $R_{S}$, is always used to damp Josephson oscillations occurring after the junction switching. A workable operation regime is achieved with StewardMcCumber parameter value, $\beta_{c}=\left(2 \pi / \Phi_{0}\right) j_{c} c R_{S}^{2} a^{2} \approx 1$. Corresponding, $R_{S} \approx 5 \Omega$ is commonly implemented with $\mathrm{MoN}_{x}$ having resistance approximately $5 \Omega$ per square [14]. Taking into account the interconnect area with minimum wiring feature size (ca. $0.5-1 \mu \mathrm{m}$ ) the shunt about triples the total area of the junction. The parallel combination of the shunt and the internal resistance provides sum-mV characteristic voltage and sub-THz characteristic frequency of the junction.

A natural approach to scale the junction area below micron size is to increase the critical-current density. Starting from $j_{c}=0.5 \mathrm{~mA} / \mu \mathrm{m}^{2}$ the junction becomes self-shunted $[15,16]$. For example, the most functionally dense RAM circuit fabricated recently [17] was based on the Josephson junctions having $j_{c}=0.6 \mathrm{~mA} / \mu \mathrm{m}^{2}$ with no shunt resistors [16], while minimum junction area was about $a \approx 0.2 \mu \mathrm{m}^{2}\left(\beta_{c} \approx 2\right)$. However, the criticalcurrent-density increase corresponds to the decrease of tunneling barrier thickness and according increase of its inhomogeneity in coordinate and momentum space. The influence of fluctuations in $\mathrm{AlO}_{x}$ transparency (caused, e.g., by pin holes and localized states) on the spread of Josephson junction critical currents becomes even more pronounced with the decrease of the junctions area. Since complex circuits require the uniformity of junctions, this scaling strategy is practically limited to the junction size of a few tenths of a micron.

Double-barrier, $S-I-N-I-S$, Josephson structures were proposed to mitigate the difficulties of the scaling $[18,19]$. If the $N$-layer thickness, $d_{N}$, is much smaller then the decay length,

$$
d_{N} \ll \xi_{N}=\sqrt{\hbar D_{N} / 2 \pi k_{B} T_{c}}
$$

(dirty limit), where $T_{c}$ is the superconductor critical temperature, $D_{N}=v_{F} l / 3$ is the diffusion coefficient, $v_{F}$ is the Fermi velocity and $l$ is the electron mean free path in the normal metal, then the cotunneling across the $I-N-I$ weak link may prevail over the sequential $S-I-N+N-I-S$ tunneling process resulting in $I_{c} R_{N}$ product even larger than that of the $S-I-S$ junction with the same $I$ barrier [20-22]. Simple estimations show [18] that even the formation of small pin holes of a diameter $d_{\mathrm{PH}} \ll \xi_{N}, d_{N}$, will not lead to a large spread of the junction parameters if $\sqrt{d_{\mathrm{PH}} \xi_{N}} \ll d_{N}$.

Unfortunately, due to difference in $\mathrm{Al}$ growth over $\mathrm{Nb}$ and $\mathrm{AlO}_{x}$ surfaces, the implementation of equal barriers in $\mathrm{Nb} / \mathrm{AlO}_{x} / \mathrm{Al} / \mathrm{AlO}_{x} / \mathrm{Nb}$ structure appears to be an intractable task with an increase of the barriers' transparency [23,24]. The structure asymmetry leads to localization of the weak link at one of the barriers that cancelled all the benefits, making this approach impractical for scaling [24]. The implementation of the much-needed symmetry of the transparency of the tunnel layers requires the development of two different technological processes of aluminum oxidation. In the absence of such technology, the $S-I-N-I-S$ structures turned out to be useful only for implementation of Josephson voltage standards [25-27].

Another option is to artificially synthesize the interlayer material by doping semiconductor up to a degenerate state [28-40], e.g., using $\alpha$-Si, where $\mathrm{Nb}$ or $\mathrm{W}$ can be chosen as dopants. The current transport in $\mathrm{Nb} / \alpha-\mathrm{Si} / \mathrm{Nb}(S-D-$ $S$, where $D$ stands for doped semiconductor) junction is determined by elastic and inelastic resonance tunneling processes. The latter form quasi-one-dimensional channels with metallic conductivity and provide internal junction shunting. While $I_{c} R_{N}$ product of sub-millivolt level is readily attainable $[28,37,39,40]$, the inherent inhomogeneity of the barrier transparency prohibits the scaling of such $S-D-S$ structure with preservation of the junction parameters reproducibility due to the probabilistic nature of the 
formation of resonant channels for the flow of normal and superconducting currents.

At nanometer scale, where the area of Josephson junction is $a \sim 0.01 \mu \mathrm{m}^{2}$, the Josephson junction critical current should be $j_{c} \sim 10 \mathrm{~mA} / \mu \mathrm{m}^{2}$, taking into account the required critical current $I_{c}=0.1 \mathrm{~mA}$ at the working temperature $T=4.2 \mathrm{~K}$. Such high values of the critical-current density together with the considered inhomogeneity of the barriers of $S-I-S$ and $S-D-S$ junctions makes the $S-N-S$ junction the preferred type for scaling. $S-N-S$ structure obviously does not require a shunt resistor. It is useful to choose $N$ material so as to minimize the suppression of superconductivity in $S$ electrodes while maximizing the induction of superconductivity in the $N$ interlayer for high values of $I_{c} R_{N}$ product and critical current, correspondingly. For the transparent $S-N$ interface the proximity effect is described $[41,42]$ by the suppression parameter,

$$
\gamma=\frac{\rho_{S} \xi_{S}}{\rho_{N} \xi_{N}}, \xi_{S}=\sqrt{\frac{\hbar D_{S}}{2 \pi k_{B} T_{c}}},
$$

where $\xi_{S}$ is decay length in $S$ material and $\rho_{S, N}$ are normal resistivities of $S$ and $N$ materials. The desired value, $\gamma<1$, means that the amount of normal electrons diffusing per second from $N$ to $S$ is smaller than the same value of correlated electrons moving in the opposite direction. This case is commonly realized with pairs of materials like $\mathrm{Nb}$ $(S)$ and Ti, Hf or $\mathrm{Pd}_{x} \mathrm{Au}_{1-x}(N)$, where $\xi_{N} \lesssim \xi_{S} \approx 10 \mathrm{~nm}$ while resistivity of normal metals is larger or of an order of the one of niobium, $\rho_{N} \gtrsim \rho_{S} \approx 8 \mu \Omega \mathrm{cm}$ [43]. For sandwich type $S-N-S$ junction and the $N$-layer thickness larger than $\xi_{N}$, it is necessary [42] to have $\gamma \lesssim 0.1$ for achieving a high value of $I_{c} R_{N}$ product at $T / T_{c} \approx 0.5$. However, this value of suppression parameter is difficult to implement. It is, for this reason, the experimentally obtained magnitudes of characteristic voltage is rather small $[18,44-53]$. Note that a simple decrease in the $N$-layer thickness is not a solution of this problem. In addition to technological difficulties, the decrease in $N$ layer thickness leads to spatial delocalization of the weak-link region due to the depairing process in the vicinity of the interfaces $[41,54,55]$.

This problem is inherent for commonly used sandwichtype Josephson junctions. However, it can be circumvented by modification of the weak-link region geometry with corresponding redistribution of current flowing therein [56-60]. An example of such a solution is the $S N-N-N S$ junction in the form of variable thickness bridge [61-67] presented in Fig. 1. Here a high current density in the bridge is lowered due to the current spread all over the area under the electrodes. The latter is owing to the finite transparency of $S-N$ interfaces $[65,68-70]$.

Metals with relatively large decay length are preferred to obtain a high critical-current value of $S N-N-N S$ junction. Unfortunately, such metals generally possess relatively small resistivity, since $\xi_{N} \sim \sqrt{D_{N}}$ while $\rho_{N} \sim$
$1 / D_{N}$. However, if the thickness of $N$ layer is smaller than the decay length, $d_{N} \ll \xi_{N}$, then the suppression parameter is decreased [71] proportionally to their ratio,

$$
\gamma_{M}=\gamma \frac{d_{N}}{\xi_{N}}
$$

corresponding to the decreased number of normal electrons diffusing from $N$ to $S$. Thus, the $S N-N-N S$ structure size ratio, $d_{N} \ll \xi_{N} \approx L_{b}$, seems to be optimal choice to provide both high critical current and normal state resistance of the junction.

In the following, we consider $S N-N-N S$ junction with specified ratio of geometrical parameters as the promising candidate for scaling, because the decay length, $\xi_{N}$, of the broadly utilized metals, such as $\mathrm{Al}$ or $\mathrm{Cu}$, lies in the range of a few tens of nanometers.

\section{CALCULATION OF $S N-N-N S$ JUNCTION SUPERCURRENT}

Our model of the $S N-N-N S$ junction contains a normalmetal film connecting two massive superconducting electrodes of the length, $\left(L-L_{b}\right) / 2$ each, located at the distance $\pm L_{b} / 2$ from the center of this film, see Fig. 1. The total length of the junction is $L$. In the calculation of the critical current, we suppose that the condition of the dirty limit is fulfilled for all metals, the critical temperature of $N$ material is equal to zero and its width, $W$, and thickness, $d_{N}$, are much smaller than Josephson penetration depth, $\lambda_{J}$, and decay length, $\xi_{N}$, respectively.

The proximity effect in this system can be considered in the frame of Usadel equations [72], which in the $N$ film have the form,

$$
\xi_{N}^{2} \frac{\partial}{\partial x}\left(G_{N}^{2} \frac{\partial \Phi_{N}}{\partial x}\right)+\xi_{N}^{2} \frac{\partial}{\partial y}\left(G_{N}^{2} \frac{\partial \Phi_{N}}{\partial y}\right)=\omega G_{N} \Phi_{N}
$$

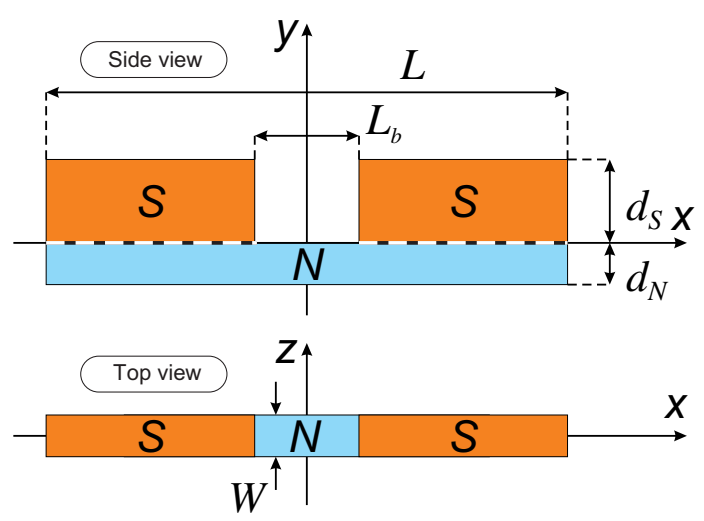

FIG. 1. Sketch of $S N-N-N S$ Josephson junction with variable thickness bridge geometry. 
Here $\Phi_{N}, G_{N}=\omega / \sqrt{\omega^{2}+\Phi_{N} \Phi_{N}^{*}}$ are modified Usadel Green's functions, and $\omega=(2 m+1) T / T_{c}$ are Matsubara frequencies, $m$ is an integer. $\Phi_{N}$, are normalized on $\pi T_{c}$.

We apply standard boundary conditions at the edges of the structure [73] and the Kupriyanov-Lukichev boundary condition [20] at $S-N$ interfaces. The conditions, $\gamma_{M} \lesssim 0.3$, $d_{N} \ll \xi_{N}$ [71], permit us to neglect the suppression of superconductivity in the $S$ film due to proximity effect even in the case of fully transparent $S$ - $N$ interfaces, and also allows considering functions $\Phi_{N}$ independent on the coordinate $y$, in the first approximation on the parameter $d_{N} / \xi_{N}$.

By integration of Eq. (4) over the coordinate $y$, we obtain the equations,

$$
\frac{\gamma_{\mathrm{BM}} \xi_{N}^{2}}{G_{N}} \frac{\partial}{\partial x}\left(G_{N}^{2} \frac{\partial \Phi_{N}}{\partial x}\right)-\left(G_{S}+\gamma_{\mathrm{BM}} \omega\right) \Phi_{N}=-G_{S} \Phi_{S}
$$

for the region of electrodes, $\left|L_{b} / 2\right| \leq|x| \leq|L / 2|$, where $\gamma_{\mathrm{BM}}=\gamma_{B} d_{N} / \xi_{N}, \gamma_{B}=R_{B} / \rho_{N} \xi_{N}$ is suppression parameter, $R_{B}$ is specific resistance of the $S-N$ interface, $\Phi_{S}$, are modified Usadel Green's functions normalized on $\pi T_{c}$, $G_{S}=\omega / \sqrt{\omega^{2}+\Phi_{S} \Phi_{S}^{*}}$. In the area of the bridge we have the following equations:

$$
\xi_{N}^{2} \frac{\partial}{\partial x}\left(G_{N}^{2} \frac{\partial \Phi_{N}}{\partial x}\right)=\omega G_{N} \Phi_{N}
$$

The solution of the problem is simplified in the limit of a small gap between superconducting electrodes, $L_{b} \ll \xi_{N}$. We also suppose that in the practically interesting case, the length of superconducting electrodes is much larger than the characteristic scale, $L-L_{b} \gg \zeta$, which arise in the frame of analytical solution of the problem [73]. This characteristic scale is $\zeta \approx \sqrt{\gamma_{\mathrm{BM}}} \xi_{N}$ in the limit of small $\gamma_{\mathrm{BM}}$ for arbitrary normalized temperature, $t=T / T_{c}$. In the opposite limit of large $\gamma_{\mathrm{BM}}$, it is $\zeta \approx \xi_{N}$ at small temperatures and $\zeta \lesssim \xi_{N}$ at $t \gtrsim 0.5$.

Under these assumptions, we obtain [73] the following expression for the product of superconducting current, $J_{S}$, across the junction and its normal state resistance, $R_{N}$,

$$
\frac{e R_{N} J_{S}}{2 \pi k_{B} T_{c}}=t \sum_{\omega \geq 0} \frac{2 \operatorname{Re} \Phi_{N}(1+2 \eta)}{\sqrt{\omega^{2}+\left(\operatorname{Re} \Phi_{N}\right)^{2}}} C
$$

where $\eta=\sqrt{\gamma_{\mathrm{BM}}} \xi_{N} / L_{b}, C$ are the constants determined by matching solutions in the ranges of electrodes and the bridge [73], and $\operatorname{Re} \Phi_{N}$ is the real part of the functions $\Phi_{N}$, while

$$
R_{N}=R_{N B}+2 R_{S N}
$$

is the sum [74] of the resistance of the bridge, $R_{N B}$, and the resistances of two $S-N$ interfaces, $R_{S N}$,

$$
R_{N B}=\frac{\rho_{N} L_{b}}{d_{N} W}, R_{S N}=\frac{\rho_{N} \xi_{N} \sqrt{\gamma_{\mathrm{BM}}}}{d_{N} W} .
$$

In the limit of small suppression parameter,

$$
\sqrt{\gamma_{\mathrm{BM}}} \ll L_{b} / \xi_{N} \ll 1
$$

(rigid boundary conditions), Eq. (7) for supercurrent takes the following form:

$$
\frac{e R_{N} J_{S}}{2 \pi k_{B} T_{c}}=t \sum_{\omega \geq 0} \frac{2 \Delta \cos \frac{\varphi}{2}}{\Omega_{1}} \arctan \frac{\Delta \sin \frac{\varphi}{2}}{\Omega_{1}},
$$

where $R_{N}=R_{N B}, \Delta$ is the magnitude of superconducting order parameter normalized on $\pi T_{c}, \varphi$ is superconducting phase difference across the junction, and

$$
\Omega_{1}=\sqrt{\Omega^{2}+\Delta^{2} \cos ^{2} \frac{\varphi}{2}}, \Omega=\omega\left(1+\gamma_{\mathrm{BM}} \sqrt{\omega^{2}+\Delta^{2}}\right) .
$$

For a vanishingly small suppression, $\gamma_{\mathrm{BM}} \rightarrow 0$, Eq. (9) transforms into the formula obtained by Kulik and Omelyanchuk (KO-1) [75].

In the opposite limit of large $\gamma_{\mathrm{BM}}$,

$$
\frac{L_{b}}{\xi_{N}} \ll \frac{\gamma_{\mathrm{BM}}}{\left(1+\gamma_{\mathrm{BM}}\right)},
$$

Eq. (7) transforms into

$$
\frac{e R_{N} J_{S}}{2 \pi k_{B} T_{c}}=t \sum_{\omega \geq 0} \frac{\sqrt{2} \Delta^{2} \sin \varphi}{\Omega_{1} \sqrt{\left(\sqrt{\Omega^{2}+\Delta^{2}}+\Omega_{1}\right) \sqrt{\omega^{2}+\Delta^{2}}}}
$$

where $R_{N}$ is mainly determined by the resistance of $S-N$ interfaces.

\section{IV. $I_{c} R_{N}$ PRODUCT OF $S N-N$-NS JUNCTION}

Characteristic voltage of the $S N-N-N S$ junction can be obtained directly from Eq. (7) and its limiting cases Eqs. (9) and (10). Figure 2(a) shows normalized $I_{c} R_{N}$ product, $v_{c}=e I_{c} R_{N} / 2 \pi k_{B} T_{c}$ (where $I_{c}=\max \left[J_{S}(\varphi)\right]$ ), as a function of normalized temperature, $t$. The normalizing coefficient value is $2 \pi k_{B} T_{c} / e \approx 5 \mathrm{mV}$ for niobium critical temperature, $T_{c}=9.2 \mathrm{~K}$. The bridge length is $L_{b}=0.1 \xi_{N}$. The curves obtained in both limits are well consistent. The presented dependencies are limited from above by the curve obtained using the KO-1 expression [75] for $\gamma_{\mathrm{BM}}=$ 0 as expected. The temperature corresponding to characteristic voltage drop by $20 \%$ from its maximum value 

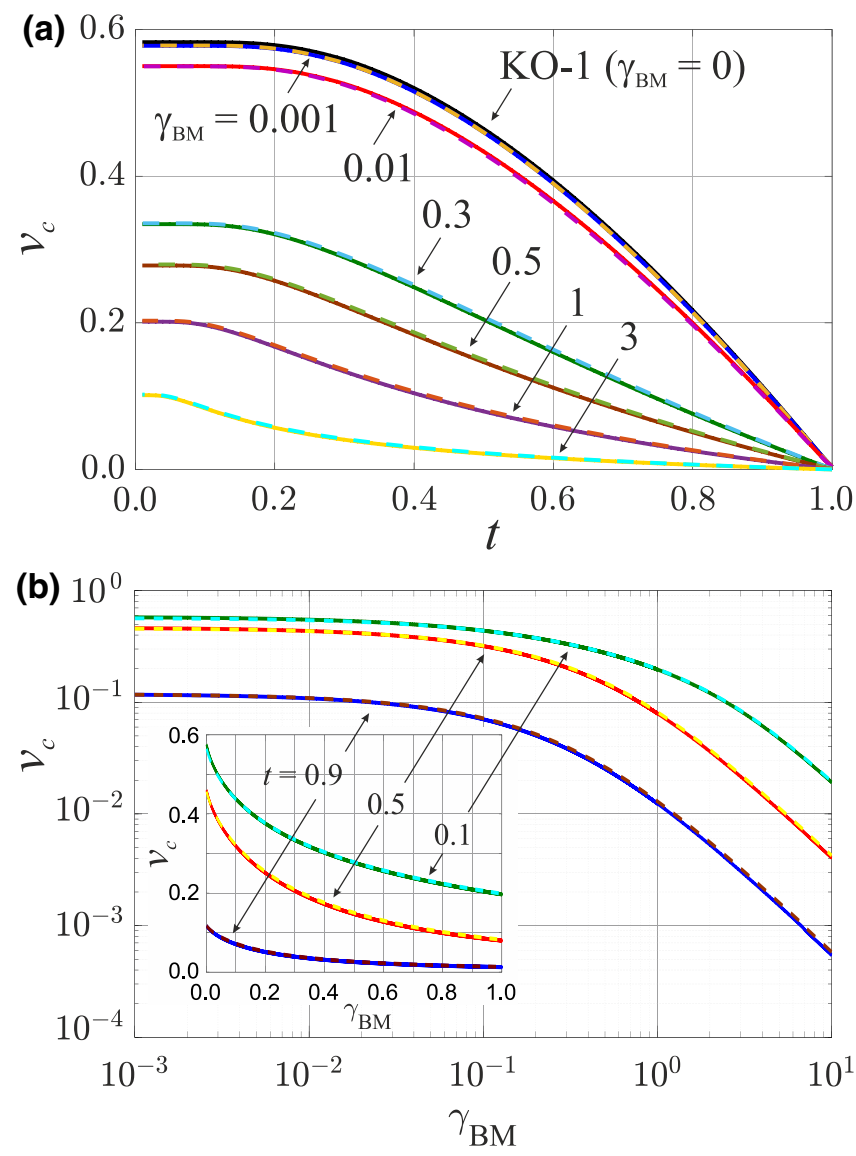

FIG. 2. Normalized $I_{c} R_{N}$ product, $v_{c}=e I_{c} R_{N} / 2 \pi k_{B} T_{c}$, of the $S N-N-N S$ Josephson junction in dependence on (a) normalized temperature, $t$, and (b) suppression parameter, $\gamma_{\mathrm{BM}}$, calculated using Eq. (7) (solid lines), (9) [dotted lines, $\gamma_{\mathrm{BM}}=0.001,0.01$, (a)] and Eq. (10) [dotted lines, $\gamma_{\mathrm{BM}}=0.3 \cdots 3$, (a) and (b)]. Inset shows $v_{c}\left(\gamma_{\mathrm{BM}}\right)$ for $\gamma_{\mathrm{BM}} \leq 1$. The upper solid line in (a) corresponds to the KO-1 expression [75].

decreases from $t \approx 0.5$ at small suppression, $\gamma_{\mathrm{BM}} \leq 0.01$, to $t \leq 0.2$ at $\gamma_{\mathrm{BM}} \geq 1$.

Figure 2(b) presents $v_{c}\left(\gamma_{\mathrm{BM}}\right)$ dependence for different temperatures in double logarithmic scale. The inset shows these dependencies for $\gamma_{\mathrm{BM}} \leq 1$. The characteristic voltage increases with the temperature decrease, in accordance with Fig. 2(a). For the commonly used temperature, $t \approx$ 0.5 , the characteristic voltage drops from its maximum value, $v_{c \max } \approx 0.46$, by $20 \%$ at $\gamma_{\mathrm{BM}} \approx 0.06$, and becomes halved at $\gamma_{\mathrm{BM}} \approx 0.25$. Starting from $\gamma_{\mathrm{BM}} \approx 1$, the characteristic voltage $v_{c}\left(\gamma_{\mathrm{BM}}\right)$ falls as $\gamma_{\mathrm{BM}}^{-3 / 2}$ with $v_{c}<0.1$. The decrease of the characteristic voltage with the suppression parameter increase is slower with the temperature decrease.

In the considered approximation, the characteristic voltage is nearly independent on the length of the bridge up to $L_{b} \lesssim \xi_{N}$ for arbitrary $\gamma_{\mathrm{BM}}$. The critical current, $I_{c}$, is the larger the smaller are $L_{b}$ and $\gamma_{\mathrm{BM}}$. The current flowing through the junction $\left(I \approx I_{c}\right)$ must not destroy the superconductivity in its electrodes. Below, we formulate the restriction on geometrical parameters of $S N-N-N S$ structure coming from this requirement.

\section{LIMITATION ON THE CURRENT IN SUPERCONDUCTING ELECTRODES}

In accordance with experimental data obtained in MIT LL [12], a Nb strip with thickness, $d_{S}=200 \mathrm{~nm}$, and width, $W=250 \mathrm{~nm}$, possesses the critical current density, $J_{\text {cst }} \approx 4.5 \times 10^{7} \mathrm{~A} / \mathrm{cm}^{2}$ so that the critical current is $I_{\text {cst }} \approx 22.5 \mathrm{~mA}$ at $T=4.2 \mathrm{~K}$. With an increase of the width, the critical current grows proportional to $\sqrt{W}$, while with $W$ decrease below $250 \mathrm{~nm}, I_{\text {cst }}$ falls linearly with $W$,

$$
I_{\mathrm{cst}}=J_{\mathrm{cst}}\left(W-W_{0}\right) d_{S},
$$

where $W_{0} \approx 50 \mathrm{~nm}$ is a doubled thickness of the contaminated surface layer.

The linear dependence of the critical current on the width means that in the range of widths that are interesting for us, $W \lesssim 250 \mathrm{~nm}$, the supercurrent is distributed uniformly over the film width. This fact allows us to seek the solution of the Usadel equations in the depth of the electrodes $(|x| \lesssim L)$ in the form $\Phi_{S, N}(x, y)=$ $\Phi_{S, N}(y) \exp \{i k x\}$ independent on coordinate $z$ (see Fig. 1). Here $k$ is an independent on $\omega$ and $x$ constant, which is proportional to superfluid velocity. By assuming further that the supercurrent flowing through the $S-N$ electrodes is significantly less than the depairing current, we find functions $\Phi_{S, N}(x)$ from the solution of the proximity effect problem between superconducting and thin normal films,

$$
\Phi_{S}(x)=\Delta, \Phi_{N}(x)=\frac{G_{S} \Delta}{G_{S}+\omega \gamma_{\mathrm{BM}}}, G_{S}=\frac{\omega}{\sqrt{\omega^{2}+\Delta^{2}}} .
$$

For supercurrent densities, $J_{S E}$ and $J_{N E}$, in the $S$ and $N$ films we get

$$
\begin{gathered}
\frac{e \rho_{S} J_{S E}}{2 \pi k_{B} T_{c}}=k S_{1}, S_{1}=t \sum_{\omega=0}^{\infty} \frac{\Delta^{2}}{\omega^{2}+\Delta^{2}}, \\
\frac{e \rho_{N} J_{N E}}{2 \pi k_{B} T_{c}}=k S_{2}, S_{2}=t \sum_{\omega=0}^{\infty} \frac{\Delta^{2}}{\omega^{2}\left(1+\omega \gamma_{\mathrm{BM}} G_{S}^{-1}\right)^{2}+\Delta^{2}},
\end{gathered}
$$

where parameter $k$ in Eqs. (12), (13),

$$
k=\frac{e \rho_{S} I_{S N}}{2 \pi T_{c} d_{S} W S_{1}(1+q)},
$$

is determined by the magnitude of the full current, $I_{S N}=$ $W\left(J_{S E} d_{S}+J_{N E} d_{N}\right)$, flowing through the $S-N$ electrodes, 
while $q$ in Eq. (14) is the ratio of the currents flowing through the $N$ and $S$ films,

$$
q=\frac{J_{N E} d_{N}}{J_{S E} d_{S}}=\frac{d_{N} \rho_{S}}{d_{S} \rho_{N}} \frac{S_{2}}{S_{1}},
$$

so that $1+q=I_{S N} / J_{S E} d_{S} W$.

At $T \approx 0.5 T_{c}$, the Matsubara frequencies $\omega$ are larger than $\Delta$. We can neglect $\Delta$ in comparison with $\omega$ when estimating the magnitudes of sums $S_{1,2}$ in Eq. (15) and get,

$$
q=\frac{d_{N} \rho_{S}}{d_{S} \rho_{N}\left(1+\gamma_{\mathrm{BM}}\right)^{2}} .
$$

The current flowing through the $S$ film must be small in comparison with $S$-film critical current,

$$
\frac{I_{c}}{I_{\mathrm{cst}}} \frac{J_{S E} d_{S} W}{I_{S N}}=\frac{I_{c}}{(1+q) I_{\mathrm{cst}}} \lesssim \beta,
$$

where $\beta \ll 1$ is the desired smallness coefficient. The magnitude of $I_{c}$ in Eq. (16) can be obtained from the data presented in Fig. $2, I_{c}=v_{c} 2 \pi k_{B} T_{c} / e R_{N}$. Since $I_{c} \sim R_{N}^{-1} \sim$ $d_{N}$ and $I_{\text {cst }} \sim d_{S}$, the restriction, Eq. (16), transforms into

$$
\frac{\mathcal{C}}{1+q} \frac{d_{N}}{d_{S}} \lesssim \beta
$$

where the coefficient $\mathcal{C}$ for niobium is

$$
\mathcal{C}=\frac{v_{c} \times 5 \mathrm{mV}}{J_{\mathrm{cst}} \rho_{N}\left(L_{b}+2 \xi_{N} \sqrt{\gamma_{\mathrm{BM}}}\right)} .
$$

We should note that for $\rho_{N} \approx \rho_{S}$ the ratio of the currents flowing through the $N$ and $S$ films is small, $q \ll 1$, and so $1+q \approx 1$.

\section{HEAT BALANCE}

A requirement to the design of circuits based on the $S N$ $N-N S$ junction is that the normal component, $I_{N}$, of the total current must not lead to overheating of the $S N-N$ $N S$ structure. Here we calculate the power dissipated in the junction during a clock period of a digital circuit and the power that can be absorbed by the junction environment. Then we evaluate the heating of niobium-copper $S N-N-N S$ structure.

\section{A. Dissipated and absorbed power}

The heating effect arises due to Joule dissipation. The energy dissipated during the junction switching, $t_{\mathrm{sw}} \approx$ $\Phi_{0} / I_{c} R_{N}$, can be estimated as

$$
E=\int_{0}^{t_{\mathrm{sw}}} I_{N} V d \tau,
$$

where $V=(\hbar / 2 e) \partial \varphi / \partial \tau$ is a voltage drop across the junction and $I_{N}=V / R_{N}$. By assuming that the phase increase rate is $\partial \varphi / \partial \tau \approx 2 \pi / t_{\mathrm{sw}}$ and that the junction is switching once in a clock period, $T_{\text {clk }}$, we obtain the dissipated energy and average dissipated power,

$$
E \approx I_{c} \Phi_{0}, P=E / T_{\text {clk }}
$$

The energy $E$ is transferred to normal electrons located in the region of concentration of the electric field, which includes the bridge between $S-N$ electrodes. The hot electrons may diffuse from the heated region on the characteristic time scale $\tau_{\text {esc }} \approx L_{b}^{2} / D_{N}$. For a typical value of Fermi velocity, $v_{F} \approx 10^{6} \mathrm{~m} / \mathrm{s}$, electronic mean free path, $\ell \approx d_{N} \approx 10 \mathrm{~nm}$, and the bridge length, $L_{b} \approx \xi_{N} \approx 40 \mathrm{~nm}$, a rough estimation of $\tau_{\text {esc }}$ gives the value approximately $3 \times 10^{-13} \mathrm{~s}$. This is by an order of magnitude larger than the scattering time, $\tau_{\mathrm{sc}}=\ell / v_{F} \approx 10^{-14} \mathrm{~s}$.

Such a short value of $\tau_{\mathrm{esc}}$ means that for a time equal to a clock period lying in picosecond time scale, hot electrons diffuse along the $N$ film into the $S$ - $N$ electrodes over a distance of the order of the current leakage length, $\xi_{N} \sqrt{\gamma_{\mathrm{BM}}}$. This process is shown schematically by the red arrows in Fig. 3.

Within the standard model of electron energy relaxation, the electron-electron collision rate is assumed to be large so that the electrons maintain an equilibrium distribution characterized by the temperature, $T_{\text {loc }}$, which is different from the lattice temperature $T$. The hot electrons may relax the excess energy making use of the two channels schematically shown in Fig. 3 by yellow and blue arrows.

The first channel for relaxation is the diffusion of hot electrons into superconducting electrodes. The heat conductance of the $S-N$ interface with small specific boundary

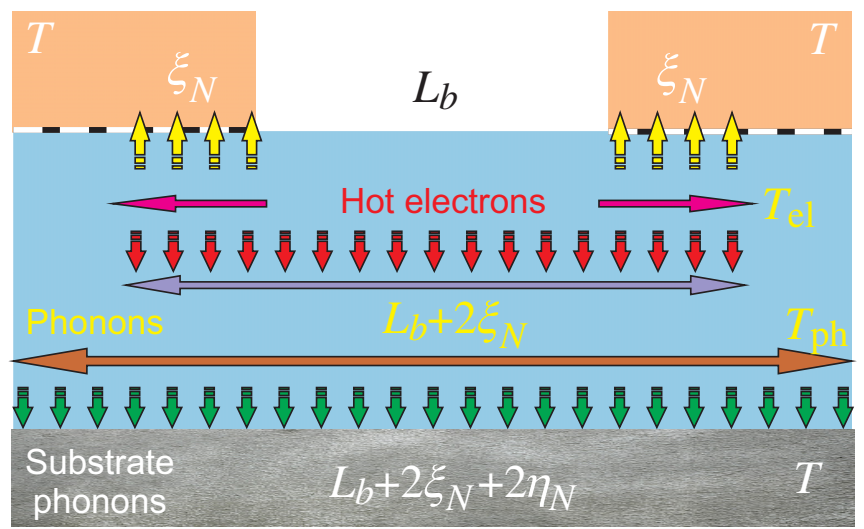

FIG. 3. Sketch of a $S N-N-N S$ Josephson junction with a variable thickness bridge geometry. The horizontal red, purple, and brown arrows indicate the areas where heat is transferred to the $S$ electrodes, phonon subsystems in the normal film and substrate, respectively. The vertical yellow and red arrows schematically indicate the process of heat transfer from hot electrons to the electrodes and the phonon subsystem of a normal film. Vertical green arrows indicate the process of heat transfer into the substrate. 
resistance, $R_{B}$, is given by the expression [76]

$$
P_{S N}=\frac{2 \Delta}{e^{2} R_{B}} A \sqrt{\frac{2 \pi \Delta}{k_{B} T_{\mathrm{loc}}}} \exp \left(-\frac{\Delta}{k_{B} T_{\mathrm{loc}}}\right) k_{B} \delta T,
$$

where $\delta T=T_{\text {loc }}-T, A$ is the effective area through which the heat flow takes place. For a finite transparency of the $S$ - $N$ interface $\left(\gamma_{\mathrm{BM}} \approx 1\right)$, the area $A \approx 2 \xi_{N} W$.

The second channel is the transfer of excess energy to phonons. In dirty metals, the rate of this process , $P_{\mathrm{e}-\mathrm{ph}}$, is given by [77]

$$
\begin{gathered}
P_{\mathrm{e}-\mathrm{ph}}=K_{*}\left(\frac{3 \zeta(5)}{3 \pi} \frac{c_{t}^{4}}{c_{l}^{4}} \frac{T_{\mathrm{loc}}^{5}}{T_{*}^{5}}+\frac{4 \pi^{2}}{45} \frac{T_{\mathrm{loc}}^{4}}{T_{*}^{4}}\right), T_{\mathrm{loc}}>T_{*}, \\
K_{*}=\frac{N_{0} \tau_{\mathrm{sc}} k_{B} T_{*}}{d_{f n} \ell^{5}}\left(\frac{p_{F}^{2}}{m_{e}}\right)^{2}, T_{*}=\frac{\hbar c_{t}}{k_{B} \ell}
\end{gathered}
$$

where $N_{0}$ is the density of states at Fermi level, $c_{l}, c_{t}$, are longitudinal and transverse speeds of sound, $d_{f n}$ is the mass density, $p_{F}$ is Fermi momentum, $m_{e}$ is electron mass, $\zeta(z)$ is the Riemann $\zeta$ function. Expression (22) can be rewritten via the thermal conductance per unit volume $K(T)=d P_{\mathrm{e}-\mathrm{ph}} / d T_{\mathrm{loc}}$

$$
P_{\mathrm{e}-\mathrm{ph}}=\frac{K_{*}}{T_{*}}\left(\frac{15 \zeta(5)}{3 \pi} \frac{c_{t}^{4}}{c_{l}^{4}} \frac{T_{\mathrm{loc}}^{4}}{T_{*}^{4}}+\frac{16 \pi^{2}}{45} \frac{T_{\mathrm{loc}}^{3}}{T_{*}^{3}}\right) \delta T_{\mathrm{e}-\mathrm{ph}},
$$

where $\delta T_{\mathrm{e} \text {-ph }}=T_{\mathrm{loc}}-T_{\mathrm{ph}}, T_{\mathrm{ph}}$, is the local temperature of phonon subsystem in the heated segment.

The process of energy transformation from hot electrons to phonons occurs approximately in a volume $\left(L_{b}+2 \xi_{N}\right) d_{N} W$ marked by a violet arrow in Fig. 3 .

Further phonon heat propagation along the $N$ film can be described similar to the heat spread in an infinite rod from the inner heated segment, $-\xi_{N}-L_{b} / 2 \leq x \leq \xi_{N}+L_{b} / 2$, which has a temperature $T_{\text {loc }}$ exceeding the temperature $T$ of its ends in the initial moment,

$$
\begin{aligned}
\delta T_{\mathrm{e}-\mathrm{ph}}(x, \tau)= & \frac{\delta T_{\mathrm{e}-\mathrm{ph}}(x, 0)}{2}\left[\operatorname{erf}\left(\frac{x+\xi_{N}+L_{b} / 2}{2 \sqrt{\mu_{N}^{2} \tau}}\right)\right. \\
& \left.-\operatorname{erf}\left(\frac{x-\xi_{N}-L_{b} / 2}{2 \sqrt{\mu_{N}^{2} \tau}}\right)\right],
\end{aligned}
$$

where $\operatorname{erf}(z)$ is the error function, $\mu_{N}^{2}=\lambda_{N} / C_{v n} d_{f n}, \lambda_{N}$ and $C_{v n}$ are thermal conductivity and thermal capacity of the $N$ metal.
During a clock period, $\delta T_{\mathrm{e}-\mathrm{ph}}$ decreases from its initial value down to

$$
\delta T_{\mathrm{e}-\mathrm{ph}}=\delta T_{\mathrm{e}-\mathrm{ph}}(x, 0) \operatorname{erf}\left(\frac{2 \xi_{N}+L_{b}}{4 \sqrt{\mu_{N}^{2} T_{\mathrm{clk}}}}\right) .
$$

The product $\eta_{N}=\mu_{N} \sqrt{T_{\mathrm{clk}}}$ determines the characteristic scale of the heat propagation.

The excess phonon temperature may further relax to the substrate (see green arrows in Fig. 3). The power transfer from the phonons in the normal metal to the substrate is given by the Kapitza coupling [78]

$$
P_{K}\left(T_{N} ; T\right)=K_{k} A_{\mathrm{ph}}\left(T_{\mathrm{ph}}^{4}(x, t)-T^{4}\right) \approx 4 K_{k} A_{\mathrm{ph}} T^{3} \delta T_{\mathrm{ph}-\mathrm{s}},
$$

where $\delta T_{\mathrm{ph}-\mathrm{s}}=T_{\mathrm{ph}}-T, K_{k}$ depends on the materials, and $A_{\mathrm{ph}} \approx\left(L_{b}+2 \xi_{N}+2 \eta_{N}\right) W$ is the effective interface area of the heat transfer marked by the brown arrow in Fig. 3.

\section{B. Heating of $S N-N-N S$ structures based on $\mathrm{Nb} / \mathrm{Cu}$ material combination}

The $S N-N-N S$ variable thickness bridge proposed in this work can be fabricated using commonly used materials like $\mathrm{Nb}, \mathrm{MoRe}, \mathrm{V}$ as a superconductor and $\mathrm{Cu}, \mathrm{Au}, \mathrm{Al}$ as a normal metal. Below, we estimate the parameters of $\mathrm{NbCu}$ $\mathrm{Cu}-\mathrm{CuNb}$ structure in accordance with the restriction, Eq. (17), and then calculate its heating assuming the clock period, $T_{\text {clk }}=40 \mathrm{ps}$ (clock frequency, $f_{\mathrm{clk}}=25 \mathrm{GHz}$ ).

Substitution of the typical values [79] of normal resistivity $\rho_{N}=3.7 \mu \Omega \mathrm{cm}$, decay length $\xi_{N}=37 \mathrm{~nm}$ for $\mathrm{Cu}$ and $\rho_{S}=8 \mu \Omega \mathrm{cm}$ for $\mathrm{Nb}$ into the obtained restriction on the critical current, Eq. (17), shows that the inequality can be fulfilled already for $\gamma_{\mathrm{BM}} \geq 0.2$ at $T \approx 0.5 T_{c}$ with $\beta=0.05$ if $L_{b}=\xi_{N}, d_{N}=10 \mathrm{~nm}$, and $d_{S} \gtrsim 200 \mathrm{~nm}$. Here $\gamma_{B}=\gamma_{\mathrm{BM}} \xi_{N} / d_{N} \approx 1$ is naturally implemented at the interface of the considered materials.

For $\gamma_{\mathrm{BM}}=0.5$ we obtain $v_{c} \approx 0.14$ (see Fig. 2) so that $I_{c} R_{N}=0.7 \mathrm{mV}, I_{c} \approx 0.53 \mathrm{~mA}$, and $R_{N} \approx 1.3 \Omega$. The dissipated energy during a clock period is $E=I_{c} \Phi_{0} \approx 1.1 \mathrm{aJ}$ and therefore the dissipated power is

$$
P=E / T_{\mathrm{clk}} \approx 2.7 \times 10^{-8} \mathrm{~W} .
$$

The specific boundary resistance is $R_{B}=\gamma_{\mathrm{BM}} \rho_{N} \xi_{N}^{2} / d_{N} \approx$ $2.5 \times 10^{-15} \Omega \mathrm{m}^{2}$. By putting further $T_{\mathrm{loc}} \approx T=4.2 \mathrm{~K}$ and $\Delta \approx 1.69 k_{B} T_{c}$ in Eq. (21), we get the power flowing to the $S$ films across $S-N$ interfaces,

$$
P_{S N}=K_{S N} \delta T,
$$

where $K_{S N} \approx 2 \times 10^{-7} \mathrm{~W} / \mathrm{K}$. 
By substituting the typical $\mathrm{Cu}$ parameters, $v_{F}=1.57 \times$ $10^{6} \mathrm{~m} / \mathrm{s}, d_{f n}=8900 \mathrm{~kg} / \mathrm{m}^{3}, c_{t}=4.8 \mathrm{~km} / \mathrm{s}, c_{l}=2.3 \mathrm{~km} / \mathrm{s}$, $T_{*}=1.8 \mathrm{~K}, \ell=10 \mathrm{~nm}, m_{e}=9.1 \times 10^{-31} \mathrm{~kg}, E_{F}=$ $1.13 \times 10^{-18} \mathrm{~J}$ into Eq. (24) and taking into account that the energy exchange between hot electrons and phonons occurs inside the volume of the order of $\left(L_{b}+2 \xi_{N}\right) d_{N} W$, we get the power transfer to phonons,

$$
P_{\mathrm{e}-\mathrm{ph}}=K_{\mathrm{e}-\mathrm{ph}} \delta T_{\mathrm{e}-\mathrm{ph}},
$$

where $K_{\mathrm{e}-\mathrm{ph}} \approx 1.9 \times 10^{-9} \mathrm{~W} / \mathrm{K}$.

Further power transfer between the normal metal and the substrate phonons is given by the Kapitza coupling, Eq. (27). The coefficient $K_{k}$ at the $\mathrm{Cu} / \mathrm{Si}$ interface approximately equals [80-82] to $100 \mathrm{~W} \mathrm{~m}^{-2} \mathrm{~K}^{-4}$. By taking [83-85] the thermal conductivity, $\lambda_{N} \approx 500 \mathrm{~W} / \mathrm{m} \mathrm{K}$, and thermal capacity, $C_{v n} \approx 0.3 \mathrm{~J} / \mathrm{kg}$, for $\mathrm{Cu}$, we get $\eta_{N} \approx 70$ $\mathrm{nm}$ and Eq. (27) reads

$$
P_{k}=K_{k k} \delta T_{\mathrm{ph}-\mathrm{s}},
$$

where $K_{k k} \approx 1.5 \times 10^{-9} \mathrm{~W} / \mathrm{K}$.

In accordance with the heat balance described in the previous section, the power dissipated in the junction can be absorbed by the $S$ electrodes and the phonons of the $N$ film with subsequent partial power transfer into the substrate. Since $K_{k k} \approx K_{\mathrm{e}-\mathrm{ph}} \ll K_{S N}$, one can estimate the heating of the structure as follows:

$$
\delta T=\frac{P}{K_{S N}} \approx 0.13 \mathrm{~K} .
$$

Such temperature increase can lead to a slight decrease in the value of the critical current by the amount

$$
\left|\frac{\delta I_{c}}{I_{c}}\right|=\left|\frac{\partial v_{c}}{v_{c} \partial t}\right| \frac{\delta T}{T_{c}} \approx 3.5 \%,
$$

see Fig. 2(a). The obtained deviation of the critical current seems acceptable in the view of standard optimization of the critical currents of Josephson junctions in digital circuits within the margins $\pm 20 \%$ and standard technological critical-current spread, $\delta I_{\text {ctec }} \approx 3 \%$.

The estimations, Eqs. (32), (33), show that in the temperature range of interest, $T \approx 0.5 T_{c}$, hot electrons effectively diffuse from the weak-link region into massive superconducting films, thereby eliminating the effect of the nonequilibrium state of the electronic subsystem on the mode of operation of $S N-N-N S$ junction. It is necessary to mention that the lowering of the operation temperature leads to exponential suppression of the heat-transfer channel from hot electrons to $S$ electrodes. This can lead to the noticeable difference between electron and phonon temperatures, especially in $\mathrm{mK}$ temperature range.

\section{DISCUSSION}

In summary, we argue that $S N-N-N S$ junction with variable thickness bridge geometry is promising for miniaturization. The presented theoretical analysis shows that the junction $I_{c} R_{N}$ value reaches submillivolt level even when the rigid boundary conditions are not fulfilled at the $S-N$ interface. According to the obtained analytical expressions, the junction current-phase relation is close to a sinusoidal one at the operation temperature, $T / T_{c} \approx 0.5$. This allows the use of standard computer-aided design tools for superconducting digital circuit simulations.

The suppression parameter value, $\gamma_{\mathrm{BM}} \approx 1$, can be taken as the upper threshold for the fabrication of Josephson junctions intended for operation at liquid-helium temperature, $T \approx 4.2 \mathrm{~K}$. The corresponding normalized characteristic voltage, $v_{c} \approx 0.08$, turns into $I_{c} R_{N} \approx 0.4 \mathrm{mV}$ and characteristic frequency, $I_{c} R_{N} / \Phi_{0} \approx 200 \mathrm{GHz}$. Since the clock frequency of complex circuits is usually a fraction of the characteristic one, the chosen value of $\gamma_{\mathrm{BM}} \lesssim 1$ provides the possibility of operation with frequencies up to several tens of GHz.

Based on available experimental data [12] we argue that the optimal width of $S N-N-N S$ structure lays nearby 250 $\mathrm{nm}$. For a pair of materials like $\mathrm{Nb}$ and $\mathrm{Cu}$, for $W=250$ $\mathrm{nm}, d_{N}=10 \mathrm{~nm}$, and $L_{b}=40 \mathrm{~nm}$, the characteristic voltage, $I_{c} R_{N} \approx 0.7 \mathrm{mV}$, is achieved for $I_{c} \approx 0.53 \mathrm{~mA}$ and $R_{N} \approx 1.3 \Omega$.

An increase of $W$ leads to undesirable inhomogeneity of the supercurrent distribution in the $S$ electrodes. Its decrease results in the fast suppression of the $S$-film critical current. The use of additional technological steps aimed at the decrease of $S-N$ interface transparency may be required to decrease the junction critical-current density in this case.

One of the possible ways to controllably reduce the $S$ $N$ interface transparency is the technique that has proved itself in the fabrication of $\mathrm{Nb} / \mathrm{Al}-\mathrm{AlO}_{x} / \mathrm{Nb}$ tunnel junctions $[3,13,15,16]$. Here a $\mathrm{Nb} / \mathrm{Al}$ sandwich can be used as a normal metal. A thin nonsuperconducting $\mathrm{Nb}$ film plays the role of a buffer layer. The subsequent growth of $\mathrm{Al}$ on such a buffer can contribute to the formation of an atomically smooth free surface of $\mathrm{Al}$, which provides predominantly mirror reflection of conducting electrons. The required $\mathrm{Nb} / \mathrm{Al}$ boundary transparency, $\gamma_{B}$, can be achieved either naturally $[68,86]$ or by using the aluminum oxidation regime previously used in the fabrication of high- $J_{c}$ tunnel junctions [24]. Contrary to double barrier devices, in $S N-N-N S$ junctions the two $S-N$ interfaces are oxidized under the same conditions thus keeping the symmetry of the structure.

The decrease of the transparency of the $S-N$ interface corresponds to a decrease in the characteristic voltage in proportion to $\gamma_{\mathrm{BM}}^{-3 / 2}$ and in the critical current in proportion to $\gamma_{\mathrm{BM}}^{-2}$. Ultimately, the width limitations is determined by the quality of the edges of the superconducting 
electrodes. In the presence of "dead" edges approximately $25 \mathrm{~nm}$ wide, the width can hardly be less than $150 \mathrm{~nm}$.

An effective area of the $S N-N-N S$ junction, $a_{J J}$, can be determined according to the length within which the dominating heat transfer from hot electrons to superconducting films takes place, $L_{\mathrm{HT}}=L_{b}+2 \xi_{N}$. Thus $a_{J J}=3 \xi_{N} W$ for $L_{b}=\xi_{N}$ so that with $W=150 \mathrm{~nm}$ we obtain $a_{J J} \approx$ $18000 \mathrm{~nm}^{2}$. This is close to the area of semiconductor transistor, $a_{t}$, fabricated in the frame of a 40-nm technological process, under the assumption that $a_{t} \approx 50 \lambda^{2}$, where $\lambda$ is the minimum feature size [87].

\section{ACKNOWLEDGMENTS}

The authors are grateful to D.V. Averin for fruitful discussions. The work is supported by Grant No. 20-12-00130 of the Russian Science Foundation. M.Yu.K. and N.V.K. are grateful to the Interdisciplinary Scientific-Educational School of the Moscow State University "Photonic and Quantum Technologies. Digital Medicine."

[1] G. E. Moore, in IEEE International Solid-State Circuits Conference, 2003. Digest of Technical Papers. ISSCC (2003).

[2] D. S. Holmes, A. L. Ripple, and M. A. Manheimer, Energyefficient superconducting computing-power budgets and requirements, IEEE Trans. Appl. Supercond. 23, 1701610 (2013).

[3] S. K. Tolpygo, Superconductor digital electronics: Scalability and energy efficiency issues, Low Temp. Phys. 42, 361 (2016).

[4] I. I. Soloviev, N. V. Klenov, S. V. Bakurskiy, M. Y. Kupriyanov, A. L. Gudkov, and A. S. Sidorenko, Beyond Moore's technologies: Operation principles of a superconductor alternative, Beilstein J. Nanotechnol 8, 2689 (2017).

[5] M. Bhushan et al., IRDS 2020: Cryogenic Electronics and Quantum Information Processing, https://irds.ieee.org/ editions/2020 (2020), part of IEEE International Roadmap for Devices and Systems.

[6] E. P. DeBenedictis, New design principles for cold, scalable electronics, available at http://www.debenedictis.org/erik/ Cryo'FPGA'2LAL/DPfC'51.pdf.

[7] O. A. Mukhanov, 100 years of superconductivity (Taylor \& Francis: London, UK, 2011) Chap. History of Superconductor Analog-to-Digital Converters, p. 440.

[8] O. A. Mukhanov, Applied superconductivity: Handbook on devices and applications (Wiley-VCH Verlag GmbH \& Co. KGaA: Weinheim, Germany, 2015) Chap. Superconductor Digital Electronics, p. 1.

[9] O. A. Mukhanov, D. Gupta, A. M. Kadin, and V. K. Semenov, Superconductor analog-to-digital converters, Proc. IEEE 92, 1564 (2004).

[10] S. Nagasawa, H. Numata, Y. Hashimoto, and S. Tahara, High-frequency clock operation of Josephson 256-word/spl times/16-bit RAMs, IEEE Trans. Appl. Supercond. 9, 3708 (1999).
[11] Cryogenic Computing Complexity (C3), available at https://www.iarpa.gov/index.php/research-programs/c3.

[12] S. K. Tolpygo, V. Bolkhovsky, R. Rastogi, S. Zarr, E. Golden, T. J. Weir, L. M. Johnson, V. K. Semenov, and M. A. Gouker, in Applied Superconductivity Conference, ASC 2020 Virtual Conference, 49, Vol. 14 (Superconductivity News Forum (SNF), 2021), p. STP669 Wk1EOr3B-01, available: https://snf.ieeecsc.org/issues/snfissue-no-49march-2021.

[13] S. K. Tolpygo, V. Bolkhovsky, R. Rastogi, S. Zarr, A. L. Day, E. Golden, T. J. Weir, A. Wynn, and L. M. Johnson, Advanced fabrication processes for superconductor electronics: Current status and new developments, IEEE Trans. Appl. Supercond. 29, 1102513 (2019).

[14] S. K. Tolpygo, V. Bolkhovsky, T. J. Weir, A. Wynn, D. E. Oates, L. M. Johnson, and M. A. Gouker, Advanced fabrication processes for superconducting very large scale integrated circuits, IEEE Trans. Appl. Supercond. 26, 1 (2016).

[15] S. K. Tolpygo, V. Bolkhovsky, S. Zarr, T. J. Weir, A. Wynn, A. L. Day, L. M. Johnson, and M. A. Gouker, Properties of unshunted and resistively shunted $\mathrm{Nb} / \mathrm{AlOx}-\mathrm{Al} / \mathrm{Nb}$ Josephson junctions with critical current densities from 0.1 to $1 \mathrm{~mA} / \mu \mathrm{m}^{2}$, IEEE Trans. Appl. Supercond. 27, 1100815 (2017).

[16] S. K. Tolpygo, V. Bolkhovsky, D. E. Oates, R. Rastogi, S. Zarr, A. L. Day, T. J. Weir, A. Wynn, and L. M. Johnson, Superconductor electronics fabrication process with $\mathrm{MoN}_{x}$ kinetic inductors and self-shunted Josephson junctions, IEEE Trans. Appl. Supercond. 28, 1100212 (2018).

[17] V. K. Semenov, Y. A. Polyakov, and S. K. Tolpygo, Very large scale integration of Josephson-junction-based superconductor random access memories, IEEE Trans. Appl. Supercond. 29, 1302809 (2019).

[18] M. Y. Kupriyanov, A. Brinkman, A. A. Golubov, M. Siegel, and H. Rogalla, Double-barrier Josephson structures as the novel elements for superconducting large-scale integrated circuits, Phys. C 326-327, 16 (1999).

[19] A. Brinkman, D. Cassel, A. A. Golubov, M. Y. Kupriyanov, M. Siegel, and H. Rogalla, Double-barrier Josephson junctions: Theory and experiment, IEEE Trans. Appl. Supercond. 11, 1146 (2001).

[20] M. Y. Kuprianov and V. F. Lukichev, Effect of boundary transparency on critical current in dirty SS'S structures, Sov. Phys. JETP 67, 1163 (1988).

[21] A. Galaktionov and A. Zaikin, Quantum interference and supercurrent in multiple-barrier proximity structures, Phys. Rev. B 65, 184507 (2002).

[22] In RSFQ integrated circuits [88] made on the basis of double-barrier $S-I-N-I-S$ structures, the critical-current density was set to a nominal value of $j_{c}=5 \mu \mathrm{A} / \mu \mathrm{m}^{2}$, with values of the characteristic voltage equal to or larger than $160 \mu \mathrm{V}$. Such high values of the $I_{c} R_{N}$ were due to the fact that, when evaluated, the normal resistance was determined in the region of low voltages in the initial quasilinear part of the $I-V$ characteristic and was a dynamic resistance in which the magnitude exceeded at least by an order of magnitude the true normal resistance of the structure [89].

[23] F. Born, D. Cassel, K. Ilin, A. Klushin, M. Siegel, A. Brinkman, A. Golubov, M. Kupriyanov, and H. Rogalla, 
Transport properties of SINIS junctions with high-current density, IEEE Trans. Appl. Supercond. 13, 1079 (2003).

[24] S. Tolpygo, A. Brinkman, A. Golubov, and M. Kupriyanov, Transport properties of SINIS junctions with high-current density, IEEE Trans. Appl. Supercond. 13, 138 (2003).

[25] O. Kieler, R. Behr, F. Muller, H. Schulze, J. Kohlmann, and J. Niemeyer, Improved $1 \mathrm{~V}$ programmable Josephson voltage standard using SINIS junctions, Phys. C: Supercond. 372-376, Part 1, 309 (2002).

[26] R. Behr, J. M. Williams, P. Patel, T. J. B. M. Janssen, T. Funck, and M. Klonz, Synthesis of precision waveforms using a SINIS Josephson junction array, IEEE Trans. Appl. Supercond. 54, 612 (2005).

[27] F. Mueller, R. Behr, L. Palafox, J. Kohlmann, R. Wendisch, and I. Krasnopolin, Improved $10 \mathrm{~V}$ SINIS series arrays for applications in AC voltage metrology, IEEE Trans. Appl. Supercond. 17, 649 (2007).

[28] A. L. Gudkov, M. Y. Kupriyanov, and A. N. Samus, Properties of planar $\mathrm{Nb} / \alpha-\mathrm{Si} / \mathrm{Nb}$ Josephson junctions with various degrees of doping of the $\alpha$-Si layer, JETP 114, 818 (2012).

[29] A. L. Gudkov, M. Y. Kupriyanov, and K. K. Likharev, Properties of Josephson junctions with amorphous-silicon interlayers, Sov. Phys. JETP 67, 1478 (1988).

[30] V. A. Kulikov, L. V. Matveets, A. L. Gudkov, V. N. Laptev, and V. I. Makhov, A mm-wave radiometer with planar $\mathrm{Nb} / \alpha-\mathrm{Si} / \mathrm{Nb}$ Josephson junction, IEEE Trans. Magn. MAG-27, 2468 (1991).

[31] B. Baek, P. D. Dresselhaus, and S. P. Benz, Co-sputtered amorphous $\mathrm{Nb}_{x} \mathrm{Si}_{(1-x)}$ barriers for Josephson-junction circuits, IEEE Trans. Appl. Supercond. 16, 1966 (2006).

[32] B. Baek, P. D. Dresselhaus, and S. P. Benz, Thermal stability of $\mathrm{Nb} / \alpha-\mathrm{Nb}_{x} \mathrm{Si}_{1-x} / \mathrm{Nb}$ Josephson junctions, Phys. Rev. B 75, 054514 (2006).

[33] D. Olaya, B. Baek, P. D. Dresselhaus, and S. P. Benz, Highspeed $\mathrm{Nb} / \mathrm{Nb}-\mathrm{Si} / \mathrm{Nb}$ Josephson junctions for superconductive digital electronics, IEEE Trans. Appl. Supercond. 18, 1797 (2008).

[34] D. Olaya, P. D. Dresselhaus, S. P. Benz, J. Bjarnason, and E. N. Grossman, Amorphous Nb-Si barrier junctions for voltage standard and digital applications, IEEE Trans. Appl. Supercond. 19, 144 (2009).

[35] Y. Chong, S. I. Park, and K. Kim, Sputter deposition of $\mathrm{MoSi}_{2}$ film as a barrier for Nb-based Josephson junction, IEEE Trans. Appl. Supercond. 19, 238 (2009).

[36] F. Muller, T. Scheller, R. Wendisch, R. Behr, O. Kieler, L. Palafox, and J. Kohlmann, NbSi barrier junctions tuned for metrological applications up to $70 \mathrm{GHz}$ : $20 \mathrm{~V}$ arrays for programmable Josephson voltage standards, IEEE Trans. Appl. Supercond. 23, 1101005 (2013).

[37] D. Olaya, P. D. Dresselhaus, and S. P. Benz, 300-GHz operation of divider circuits using high- $j_{c} \mathrm{Nb} / \mathrm{Nb}_{x} \mathrm{Si}_{1-x} \mathrm{Nb}$ Josephson junctions, IEEE Trans. Appl. Supercond. 25, 1101005 (2015).

[38] W. Cao, H. Chen, T. Liang, J. Li, X. Kong, T. Sun, Q. Zhong, X. Wang, L. Wang, and Y. Zhong, Fabrication and characterization of SQUIDs with $\mathrm{Nb} / \mathrm{Nb}_{x} \mathrm{Sil}-x / \mathrm{Nb}$ junctions, IEEE Trans. Appl. Supercond. 28, 1602204 (2018).
[39] D. Olaya, M. Castellanos-Beltran, J. Pulecio, J. Biesecker, S. Khadem, T. Lewitt, P. Hopkins, P. Dresselhaus, and S. Benz, Planarized process for single-flux-quantum circuits with self-shunted $\mathrm{Nb} / \mathrm{Nb}_{x} \mathrm{Si}_{1-x} / \mathrm{Nb}$ Josephson junctions, IEEE Trans. Appl. Supercond. 29, 1101708 (2019).

[40] I. W. Haygood, E. R. J. Edwards, A. E. Fox, M. R. Pufall, M. L. Schneider, W. H. Rippard, P. D. Dresselhaus, and S. $\mathrm{P}$. Benz, Characterization of uniformity in $\mathrm{Nb} / \mathrm{Nb}_{x} \mathrm{Si}_{1-x} / \mathrm{Nb}$ Josephson junctions, IEEE Trans. Appl. Supercond. 29, 1103505 (2019).

[41] Z. G. Ivanov, M. Y. Kupriyanov, K. K. Likharev, S. V. Meriakri, and O. V. Snigirev, Boundary-conditions for the usadel and eilenberger equations, and properties of dirty SNS sandwich-type junctions, Sov. J. Low. Temp. Phys. 7, 274 (1981).

[42] M. Y. Kupriyanov and V. F. Lukichev, The influence of proximity effect in electrodes on the stationary properties of S-N-S Josephson structures, Sov. J. Low. Temp. Phys. 8, 526 (1982).

[43] The residual resistivity of $8 \mu \Omega \mathrm{cm}$ is obtained in our measurements of $100-\mathrm{nm}$-thick niobium film at 10 -K temperature.

[44] L. Fritzsch, M. Schubert, G. Wende, and H.-G. Meyer, Superconductor-normal metal-superconductor Josephson junctions with Ti interlayer, Appl. Phys. Lett. 73, 1583 (1998).

[45] R. Popel, D. Hagedorn, T. Weimann, F.-I. Buchholz, and J. Niemeyer, Superconductor-normal metal-superconductor process development for the fabrication of small Josephson junctions in ramp type configuration, Supercond. Sci. Technol. 13, 148 (2000).

[46] D. Hagedorn, R. Dolata, R. Popel, F.-I. Buchholz, and J. Niemeyer, Development of sub-micron SNS ramp-type Josephson junctions, IEEE Trans. Appl. Supercond. 11, 1134 (2001).

[47] M. Schubert, L. Fritzsch, G. Wende, and H.-G. Meyer, SNS junction on $\mathrm{Nb}$-Ti base for microwave circuits, IEEE Trans. Appl. Supercond. 11, 1066 (2001).

[48] D. Hagedorn, R. Dolata, F.-I. Buchholz, and J. Niemeyer, Properties of SNS Josephson junctions with HfTi interlayers, Physica C 372-376, Part 1, 7 (2002).

[49] P. D. Dresselhaus, Y. Chong, J. H. Plantenberg, and S. P. Benz, Stacked SNS Josephson junction arrays for quantum voltage standards, IEEE Trans. Appl. Supercond. 13, 930 (2003)

[50] D. Hagedorn, M. Khabipov, R. Dolata, F. I. Buchholz, and J. Niemeyer, An SNS technology process for ramp junction based digital superconducting circuits, IEEE Trans. Appl. Supercond. 13, 1096 (2003).

[51] M. Schubert, T. May, G. Wende, and H. G. Meyer, A cross-type SNS junction array for a quantum-based arbitrary waveform synthesizer, IEEE Trans. Appl. Supercond. 15, 829 (2005).

[52] D. Hagedorn, O. Kieler, R. Dolata, R. Behr, F. Muller, J. Kohlmann, and J. Niemeyer, Modified fabrication of planar sub- $\mu \mathrm{m}$ superconductor-normal metal-superconductor Josephson junctions for use in a Josephson arbitrary waveform synthesizer, IEEE Trans. Appl. Supercond. 19, 294 (2006). 
[53] J. Nagel, O. F. Kieler, T. Weimann, R. Woelbing, J. Kohlmann, A. B. Zorin, R. Kleiner, D. Koelle, and M. Kemmler, Superconducting quantum interference devices with submicron $\mathrm{Nb} / \mathrm{HfTi} / \mathrm{Nb}$ junctions for investigation of small magnetic particles, Appl. Phys. Lett. 99, 032506 (2011).

[54] A. Zubkov and M. Kupriyanov, Effect of depairing in electrodes on the stationary properties of weak links, Sov. J. Low Temp. Phys. 5, 279 (1983).

[55] M. Kupriyanov, Effect of a finite transmission of the insulating layer on the properties of SIS tunnel-junctions, JETP Lett. 56, 399 (1992).

[56] K. K. Likharev, Vortex motion and the Josephson effect in superconducting thin bridges, Zh. Eksp. Teor. Fiz. 61, 1700 (1971), [Sov. Phys. JETP 34, 906 (1972)].

[57] K. K. Likharev and L. A. Yakobson, Dynamical properties of superconducting filaments of finite length, Zh. Eksp. Teor. Fiz. 68, 1150 (1975), [Sov. Phys. JETP 41, 570-575 (1976)].

[58] K. K. Likharev, The relation $j_{s}(\varphi)$ for SNS bridges of variable thickness, Pis'ma Zh. Tekh. Fiz. 2, 29 (1976), [Sov. Tech. Phys. Lett. 2, 12 (1976)].

[59] P. Dubos, H. Courtois, B. Pannetier, F. K. Wilhelm, A. D. Zaikin, and G. Schön, Josephson critical current in a long mesoscopic S-N-S junction, Phys. Rev. B 63, 064502 (2001).

[60] P. M. Marychev and D. Y. Vodolazov, A Josephson junction based on a highly disordered superconductor/lowresistivity normal metal bilayer, Beilstein J. Nanotechnol. 11, 858 (2020).

[61] J. M. Warlaumont, J. C. Brown, T. Foxe, and R. A. Buhrman, Microwave-Enhanced Proximity Effect in Superconductor-Normal-Metal-Superconductor Microjunctions, Phys. Rev. Lett. 43, 169 (1979).

[62] R. B. van Dover, A. de Lozanne, and M. R. Beasley, Superconductor-normal-superconductor microbridges: Fabrication, electrical behavior, and modeling, J. Appl. Phys. 52, 7327 (1981).

[63] O. Liengme, P. Lerch, W. Liu, and P. Martinoli, Fabrication and electromagnetic properties of planar variablethickness S-N-S microbridges, IEEE Tran. Magn. 19, 995 (1983).

[64] H. Nakano and T. Aomine, Magnetic field dependence of critical currents in SNS bridges fabricated by direction of sputtering deposition and anodie oxidization, Jap. J. Appl. Phys. 26, 304 (1983).

[65] J. Sauvageau, R. Ono, A. Jain, K. Li, and J. Lukens, Length dependent properties of SNS microbridges, IEEE Tran. Magn. 21, 854 (1985).

[66] Y. Baryshev, A. Dmitriev, A. Krivospitsky, V. Lukichev, A. Orlikovsky, and K. Valiev, Josephson SNS microbridges fabricated by double electron beam lithography, Microelectron. Eng. 9, 385 (1989).

[67] Y. P. Baryshev, A. G. Vasil'ev, A. A. Dmitriyev, M. Y. Kupriyanov, V. F. Lukichev, I. Y. Luk'yanova, and I. S. Sokolova, Theoretical and experimental study of the Josephson effect in submicron SN-N-NS structures, Lithography Microelectronics 8, 187 (1989).

[68] A. Zehnder, P. Lerch, S. P. Zhao, T. Nussbaumer, E. C. Kirk, and H. R. Ott, Proximity effects in $\mathrm{Nb} / \mathrm{Al}-\mathrm{AlO}_{x}-\mathrm{Al} / \mathrm{Nb}$ superconducting tunneling junctions, Phys. Rev. B 59, 8875 (1999).

[69] D. V. Baxter, S. D. Steenwyk, J. Bass, and W. P. Pratt, Resistance and spin-direction memory loss at $\mathrm{Nb} / \mathrm{Cu}$ interfaces, J. Appl. Phys. 85, 4545 (1999).

[70] W. Park, D. V. Baxter, S. Steenwyk, I. Moraru, W. P. Pratt, and J. Bass, Measurement of resistance and spin-memory loss (spin relaxation) at interfaces using sputtered current perpendicular-to-plane exchange-biased spin valves, Phys. Rev. B 62, 1178 (2000).

[71] A. A. Golubov, M. Y. Kupriyanov, and V. F. Lukichev, Influence of the proximity effect in the electrodes on the stationary properties of SN-N-NS variable-thickness bridges, Soviet Microelectronics 12, 180 (1983).

[72] K. D. Usadel, Generalized Diffusion Equation for Superconducting Alloys, Phys. Rev. Lett. 25, 507 (1970).

[73] See Supplementary Material at http://link.aps.org/supple mental/10.1103/PhysRevApplied.16.044060 for details of the $S N-N-N S$ junction supercurrent calculation.

[74] T. Y. Karminskaya, A. A. Golubov, M. Y. Kupriyanov, and A. S. Sidorenko, Josephson effect in superconductor/ferromagnet structures with a complex weak-link region, Phys. Rev. B 81, 214518 (2010).

[75] I. O. Kulik and A. N. Omelyanchuk, Contribution to the microscopic theory of the Josephson effect in superconducting bridges, JETP. Lett. 21, 96 (1970).

[76] M. M. Leivo, J. P. Pekola, and D. V. Averin, Efficient peltier refrigeration by a pair of normal metal/insulator/superconductor junctions, Appl. Phys. Lett. 68, 1996 (1996).

[77] D. Nikolic, D. M. Basko, and W. Belzig, Electron cooling by phonons in superconducting proximity structures, Phys. Rev. B 102, 214514 (2020).

[78] E. T. Swartz and R. O. Pohl, Thermal boundary resistance, Rev. Mod. Phys. 61, 605 (1989).

[79] V. S. Stolyarov, T. Cren, C. Brun, I. A. Golovchanskiy, O. V. Skryabina, D. I. Kasatonov, M. M. Khapaev, M. Y. Kupriyanov, A. A. Golubov, and D. Roditchev, Expansion of a superconducting vortex core into a diffusive metal, Nat. Commun. 9, 2217 (2018).

[80] M. L. Roukes, M. R. Freeman, R. S. Germain, R. C. Richardson, and M. B. Ketchen, Hot Electrons and Energy Transport in Metals at Millikelvin Temperatures, Phys. Rev. Lett. 55, 422 (1985).

[81] F. C. Wellstood, C. Urbina, and J. Clarke, Hot-electron limitation to the sensitivity of the de superconducting quantum interference device, Appl. Phys. Lett. 54, 2599 (1989).

[82] R. L. Kautz, G. Zimmerli, and J. M. Martinis, Self-heating in the coulomb-blockade electrometer, J. Appl. Phys. 73, 2386 (1993).

[83] L. A. Novitskiy and I. Kogevnikov, Thermophysical Properties of Materials at low Temperatures. Handbook (publishing house "Mechanical engineering", Moscow, 1975).

[84] N. E. Phillips, Low-temperature heat capacity of metals, Crit. Rev. Solid State Mater. Sci. 2, 467 (1971).

[85] F. Pobell, Matter and Methods at Low Temperatures (Springer Berlin Heidelberg, New York, 2007), p. 77. 
[86] G. Brammertz, A. Poelaert, A. A. Golubov, P. Verhoeve, A. Peacock, and H. Rogalla, Generalized proximity effect model in superconducting bi- and trilayer films, J. Appl. Phys. 90, 355 (2001).

[87] M. Ansari, H. Afzali-Kusha, B. Ebrahimi, Z. Navabi, A. Afzali-Kusha, and M. Pedram, A near-threshold 7T SRAM cell with high write and read margins and low write time for sub-20nm FinFET technologies, Integr. VLSI J. 50, 91 (2015).
[88] D. Balashov, F.-I. Buchholz, H. Schulze, M. I. Khabipov, W. Kessel, and J. Niemeyer, Superconductor-insulatornormal- conductor-insulator-superconductor process development for integrated circuit applications, Supercond. Sci. Technol. 11, 1401 (1998).

[89] A. Brinkman, A. Golubov, H. Rogalla, F. Wilhelm, and M. Kupriyanov, Microscopic nonequilibrium theory of doublebarrier Josephson junctions, Phys. Rev. B 68, 224513 (2003). 